\title{
CONSTRUCTIONS OF POSITIVE COMMUTATIVE
} SEMIGROUPS ON THE PLANE, \|

\author{
REUBEN W. FARLEY \\ Department of Mathematical Sciences \\ Virginia Commonwealth University \\ Richmond, Virginia 23284 U.S.A. \\ (Received October 2, 1984 and in revised form December 10, 1984)
}

ABSTRACT. A positive semigroup is a topological semigroup containing a subsemigroup $\mathrm{N}$ isomorphic to the multiplicative semigroup of nonnegative real numbers, embedded as a closed subset of $E^{2}$ in such a way that 1 is an identity and 0 is a zero. Usina results in Farley [1] it can be shown that positive commutative semigroups on the plane constructed by the techniques given in Farley [2] cannot contain an infinite number of two dimensional groups. In this work an example of such a semigroup will be constructed which does, however, contain an infinite number of one dimensional groups. Also, some preliminary results are given here concernina what types of semilattices of idempotent elements are oossible for positive commutative semigroups on $E^{2}$. In particular, we will show that there is a unique positive commutative semigroup on $E^{2}$ which is the union of connected groups and which contains five idempotent elements. Also, we will show that such semigroups having nine idempotent elements are not unique by constructing an example of such a semigroup with nine idempotent elements whose semilattice of idempotent elements is not "symmetric" and hence which is not isomorphic to the semigroup with nine idempotent elements constructed in Farley [2].

KEY WORDS AND PHRASES. Positive semigroup, Clifford semigroup, semilattice of idempotent elements.

1980 MATHEMATICS SUBJECT CLASSIFICATION CODE. $22 A 15$.

1. INTRODUCTION.

A topological semigroup is a Hausdorff space together with a continuous, associative multiplication. The author has defined a positive semigroup to be a topological semigroup containing a subsemigroup $N$ isomorphic to the multiplicative semigroup of nonnegative real numbers, embedded as a closed subset of $E^{2}$ so that 1 is an identity and 0 is a zero [1]. Such semigroups which meet the additional requirement of being the union of groups are called positive Clifford semigroups [1]. 
In Farley [2] a method was given for constructing positive commutative semigroups on the plane as the union of $\{0\}$, an arbitrarily large number of two dimensional groups, and one dimensional groups which are bounding rays of the two dimensional groups. Since such semigroups are positive commutative $C l i f f o r d$ semigroups on $E^{2}$, they cannot contain an infinite number of two dimensional groups as shown in Farley [1]. However, in this work an example will be constructed of a positive commutative semigroup on $E^{2}$ which contains a sector of one dimensional groups, and thus contains an infinite number of one dimensional groups.

While the problem of attempting to discover what types of semilattices of idempotent elements are possible for positive commutative Clifford semiaroups on $E^{2}$ appears to be difficult to answer in general, some preliminary results are qiven in this work. Using the techniques employed in Farley [1] we will show that there is a unique (up to isomorphism) positive commutative $C l$ ifford semigroup on $E^{2}$ which is the union of connected groups and which contains five idempotent elements. This semigroup, along with its semilattice of idempotent elements will be described herein. Also, we will show that such semigroups having nine idempotent elements are not unique by constructing an example of such a semigroup having nine idempotent elements whose semilattice of idempotent elements is, in some sense, not symmetric, and hence is not isomorphic to the semigroup with nine idempotent elements constructed in Farley [2].

2. PREL IMINARIES.

An isomorphism bet:yeen two topological semigroups is a function which is both an alyebraic isomorphism and a homeomorphism. Unless otherwise indicaied, $R$ will denote a semigroup isomorphic to the multiplicative semigroup of real numbers, $N$ will denote the nonnegative members of $R$, and $P$ will denote the positive members of $R$. For $a$ topological space $X$, we will let $s=\{(x, x): x \& X\}$. If $R$ is an equivalence relation on $X, X / R$ will denote the set of equivalence classes of $X$ modulo $R$. A min thread is a closed interval [a,b] together with the usual topology and multiplication defined by $x \cdot y=\min \{x, y\}$.

3. CONSTRUCTION OF EXAMPLES.

We construct first an example of a positive commutative semigroup on $E^{2}$ which contains an infinite number of one dimensional groups.

EXAMPLE 1. Let us consider two copies of $N \times N$ denoting them as $N \times N$ and $M . M$. Also, let us form a sector of one dimensional groups by taking $N$. \{min thread\} and shrinking the base to a point. Let us call this sector $N^{\prime}$, and let the idempotent elements on $i$ ts bounding rays be denoted $e_{2}$ and $e_{3}$, with $e_{2} e_{3}=e_{3}$. Let $(a, b)_{n}$ denote an element of $N, N$, let $(a, b)_{m}$ denote an element of $M \times M$, and let $\left(p, e_{\alpha}\right)$ denote an element of $N^{\prime}$, where $e$ is an idempotent element. Let us define a multiplication on $S^{\prime}=\left[(N \times N) \cup(M \times M) \cup N^{\prime}\right]$ in the following manner. Let multiplication be coordinatewise in $N \times N, M \times M$, and $N^{\prime}$. Let us define $(a, b)_{n} \cdot\left(p, e_{\alpha}\right)=\left(b p, e_{\alpha}\right)=$ $\left(p, e_{\alpha}\right) \cdot(a, b)_{n},\left(p, e_{\alpha}\right) \cdot\left(p^{\prime}, e_{\beta}\right)=\left(p p^{\prime}, e_{\alpha_{\alpha}} e_{\beta}\right)=\left(p^{\prime}, e_{\beta}\right) \cdot\left(p, e_{r x}\right),(a, b)_{n} \cdot(c d)_{m}=$ $(\mathrm{ac}, \mathrm{bd})_{\mathrm{m}}=(\mathrm{c}, \mathrm{d})_{\mathrm{m}} \cdot(\mathrm{a}, \mathrm{b})_{\mathrm{n}}$, and $\left(\mathrm{p}, \mathrm{e}_{\mathrm{c} x}\right) \cdot(\mathrm{x}, \mathrm{y})_{\mathrm{m}}=(0, \mathrm{py})_{\mathrm{m}}=(\mathrm{x}, \mathrm{y})_{\mathrm{m}} \cdot\left(\mathrm{p}, \mathrm{e}_{\alpha}\right)$. This multiplication is easily checked to be associative, and its continuity follows from the continuity of multiplication in $N \times N, M \times M$, and $N^{\prime}$. So, with the usual topology, $S^{\prime}$ is a topological semigroup. Let us define a relation $R$ on $S^{\prime}$ in the following way. Let $\Delta \subset R$, and let $R$ be symmetric by definition. Let $\left[(a, b)_{m},(c, d)_{n}\right], R$ if and only 
if $a=c$, and $b=0=d$. Let $\left[(x, y)_{m},\left(p, e_{3}\right)\right] \varepsilon R$ if and only if $x=0$ and $y=p$. Let $\left[(s, t)_{n}\left(p, e_{2}\right)\right] \in R$ if and only if $s=0$, and $t=p$. Finally, let $\left[\left(0, e_{\alpha}\right),(0,0)_{m}\right] \in R$ and $\left[\left(0, e_{\alpha}\right),(0,0)_{n}\right] \in R$. Then, $R$ is clearly an equivalence relation and it is not difficult to check that $R$ is, in fact, a closed congruence. Thus by [2] $S=S^{1} / R$ is a topological semigroup on $E^{2}$.

It should be noted that an example of this type cannot be constructed using just one copy of $\mathrm{N} \times \mathrm{N}$ and sector of one dimensional groups since $e_{1} e_{2}$ would have to be 0 where $e_{1}=(1,0)_{n}$. So, this example is more or less minimal.

Upon investigating what types of semilattices of idempotent elements are possible for positive Clifford semigroups on the plane, we first note that the semigroup containing five idempotent elements constructed by the method employed in Example 1 or Example 3 of Farley [2] has the following semilattice of idempotent elements, where 1 denotes the element $(1,1)_{N}$, $f$ denotes $(1,1)_{\mathrm{J}}$, $e_{1}$ denotes $(1,0)_{N}=(1,0)_{\mathrm{J}}, e_{2}$ denotes $(0,1)_{N}=(0,1)_{\mathrm{J}}$, and 0 denotes $(0,0)_{N}=(0,0)_{\mathrm{J}}$ :

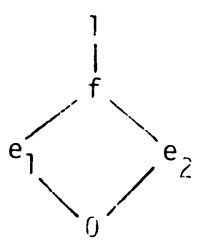

rigure 1.

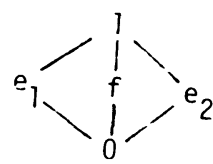

Figure 2 .

For positive Clifford semigroups on $E^{2}$ which are the union of connected groups, we will show that the semigroup is unique (up to isomorphism) among those having exactly five idempotent elements.

THEOREM 1. The positive commutative Clifford semigroup $S$ on $E^{2}$ which contains exactly five idempotent elements and is the union of connected groups is unique.

PROOF. Let $H(1)$ denote the group having the identity element 1 , and let $C L[H(1)]$ denote the closure of $\mathrm{H}(1)$. Then $\mathrm{CL}([\mathrm{H}(1)]$ is isomorphic to $\mathrm{N} N$ as shown in Farley [1], since $\mathrm{CL}[\mathrm{H}(1)]$ is two dimensional by Horne [3]. Let $e_{1}$ and $e_{2}$ denote the idempotent elements on $\mathrm{Pe}_{1}$ and $\mathrm{Pe}_{2}$, the bounding rays of $\mathrm{H}(1)$, let $f$ be the remaining idempotent element which is in $E^{2} \backslash \mathrm{CL}[H(1)]$, and let $H(f)$ be the group witri identity element $f$. Ther, the only possibilities for the semilattice of idempotent elements are the one given above ( Figure 1) and the other given in Figure 2.

We now show that this semilattice is, in fact, impossible. Since $S$ has exactly five idempotent elements and is the union of connected groups, $H(f)=E^{2} \backslash C L[H(1)]$. Now, suppose $e_{2} f=0$. Let $\left\{X_{n}\right\}$ be a sequence in $H(f)$ such that $\left\{X_{n}\right\} \cdots e_{1}$. Then $\left\{e_{1} x_{n}\right\}+e_{1}^{2}=e_{1}$. But, $e_{1} X=e_{1}(f x)$, since $f$ is the identity element in $H(f)$. So, $\left\{e_{1}\left(f x_{n}\right)\right\} \rightarrow e_{1}$. However, each term of the sequence $\left\{e_{1}\left(f x_{n}\right)\right\}$ is 0 , under the assumption that $e_{1} f=0$. This implies $\left\{e_{j}\left(f x_{n}\right)\right\} \rightarrow 0$ which is a contradiction.

A positive $\mathrm{Clifford}$ semigroup having nine idempotent elements as constructed by the method employed in Example 3 of Farley [2] has the following semilattice of idempotent elements, where 1 denotes $(1,1)_{n}, j$ denotes $(1,1)_{j}, m$ denotes $(1,1)_{m}, k$ denotes $(1,1)_{k}$, e denotes $(1,0)_{n}=(1,0)_{j}$, g denotes $(0,1)_{j}=(1,0)_{m}, h$ denotes $(0,1)_{m}=$ $(1,0)_{k}$, f denotes $(0,1)_{k}=(0,1)_{n}$ and 0 denotes $(0,0)_{m}=(0,0)_{j}=(0,0)_{n}=(0,0)_{k}:($ Fig. 3$)$ : 


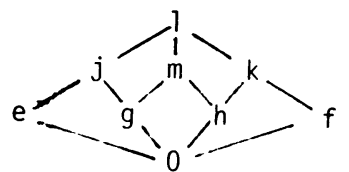

Figure 3.

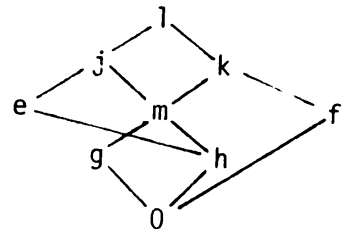

Finure 4 .

We now construct an example of a positive $C l i f f o r d$ semigroup on $E^{2}$ whose semilattice of nine idempotent elements is not isomorphic to the one above, and is, in some sense, not symmetric.

EXAMPLE 2. Let us consider four copies of $N, N$. Let us denote these copies as $N \times N$ (which will be $\vec{G}$ ), $J \quad J, M \times M$, and $K \times K$. Let us define a multiplication on $S^{\prime}=[(N \times N) \cup(J \times J) \cup(M \quad M) \cup(K, K)]$ in the following manner. Let the multiplication be coordinatewise in each copy of $N \times N$, and let the multiplication be commutative on $a l l$ of $S^{\prime}$. Let $(a, b)_{j} \cdot(c, d)_{m}=(a c, b d)_{m},(a, b)_{j} \cdot(c, d)_{k}=(a c, b c d)_{m}$, $(a, b)_{k} \cdot(c, d)_{m}=(a c, a b d)_{n, 1},(a, b)_{n} \cdot(c, d)_{j}=(a c, a b d)_{j} \cdot(a, b)_{n} \cdot(c, d)_{m}=(a c, a b d)_{m}$, and $(a, b)_{n} \cdot(c, d)_{k}=(a c, b d)_{k}$, where $(a, b)_{n} \in(N \times N),(a, b)_{m},(M \times M),(a, b)_{j}$ $(J, J)$, and $(a, b)_{k} \cdot(K \times K)$. This multiplication is easily verified to be continuous and associative. So, with the usual topology, $S^{\prime}$ is a topological semigroup. Let us define a relation $R$ on $S^{\prime}$ as follows. Let $\Delta \subset R$, and let $R$ be symetric by definition. Let $\left[(a, b)_{j},(c, d)_{n}\right], R$ if and only if $a=c$ and $b=0=d$. Let $\left[(a, b)_{j},(c, d)_{m}\right], R$ if and only if $b=d$ and $a=0=c$. Let $\left[(a, b)_{m},(c, d)_{n}\right] \in R$ if and only if $a=c$ and $b=0=d$. Finally, let $\left[(a, b)_{k},(c, d)_{n}\right] \in R$ if and only if $b=d$ and $a=0=c$. Then, $R$ is easily checked to be a closed congruence, and consequently $S^{\prime} / R$ is a topological semigroup on $E^{2}$. Let us denote by e the element $(1,0)_{n}=(1,0)_{j}$, by $g$ the element $(0,1)_{j}=(0,1)_{m}$, by $h$ the element $(1,0)_{m}=(1,0)_{k}$, and by $f$ the element $(0,1)_{k}=(0,1)_{n}$. Also, let us denote by 1 the element $(1,1)_{n}$, by $j$ the element $(1,1)_{j}$, by $m$ the element $(1,1)_{m}$, by $k$ the elment $(1,1)_{k}$, and by 0 the element $(0,0)_{n}=(0,0)_{j}=(0,0)_{m}=(0,0)_{k}$. Then, the semigroup $S=S^{\prime} / R$ has the semilattice of idemotent elements (Fig. 4$)$.

\section{REFERENCES}

1. FARLEY, R.W. Positive Clifford Semigroups on the Plane, Trans. Amer. Math. Soc. 151 (1970), 353-369.

2. FARLEY, R.W. Constructions of Positive Commutative Semigroups on the Plane, Bull. Inst. Math. Acad. Sinica ? (1979), no. 4, 357-362.

3. HORNE, J.G. Real Commutative Semigroups on the Plane, Pacific J. Math. II (1961), $981-997$.

4. HORNE, J.G. Real Commutative Semigroups on the Plane, II, Trans Amer. Math. Soc. 104 (1962), 17-23.

5. FARLEY, R.W. An Iseomorphism Theorem for Positive Commutative Semigroups on the Plane, Mat. Casopis Sloven Akad. Vied. 23 (1973), no. 2, 136-146.

6. HOFMANN, K.H., MOSTERT, P.S. Elements of Compact Semigroups, Merrill, Columbus, Ohio, 1966.

7. CLIFFORD, A.H., PRESTON, G.B. The Algebraic Theory of Semigroups, In Mathematical Surveys, vol 1, no. 7, Amer. Math. Soc., Providence, R.I., 1961.

8. MOSTERT, P.S. Plane Semigroups, Trans. Amer. Math. Soc. 103 (1962), 320-328.

9. MOSTERT, P.S., and SHIELDS, A.L. Semigroups with Identity on a Manifold, Trans. Amer. Math. Soc. 91 (1959), 380-389.

10. PONTRJAGIN, L. Topological Groups, Gittl, Moscow, 1938: English transl., Princeton Math. Series, Vol. 2, Princeton University Press, Princeton, N.J., 1939. 


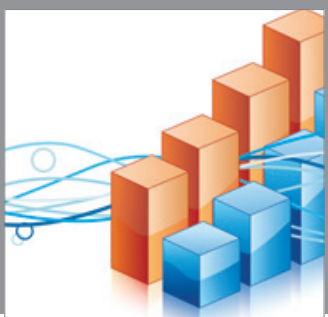

Advances in

Operations Research

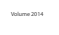

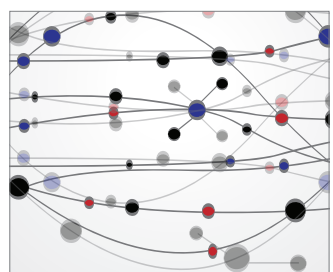

\section{The Scientific} World Journal
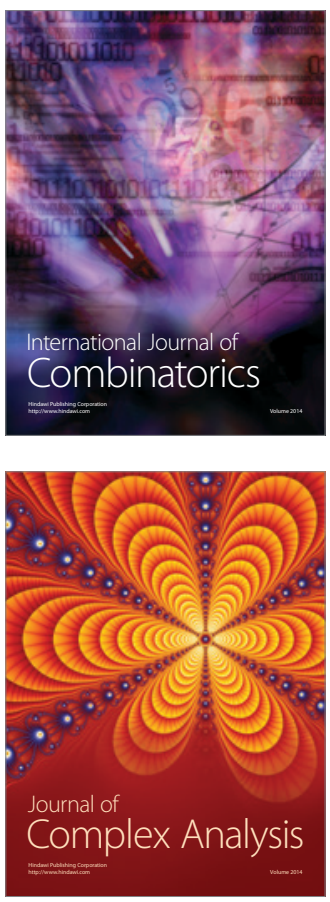

International Journal of

Mathematics and

Mathematical

Sciences
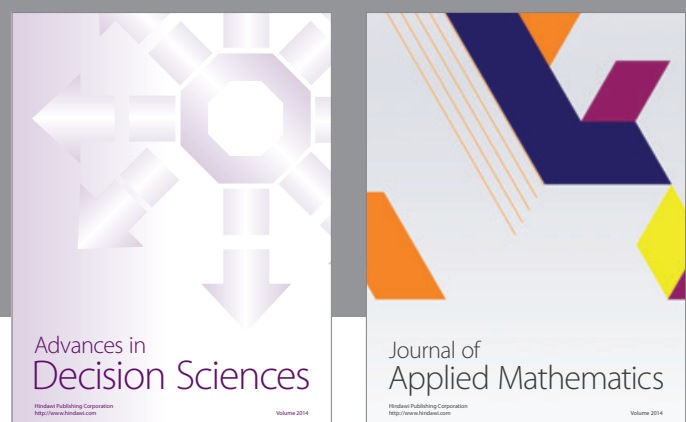

Journal of

Applied Mathematics
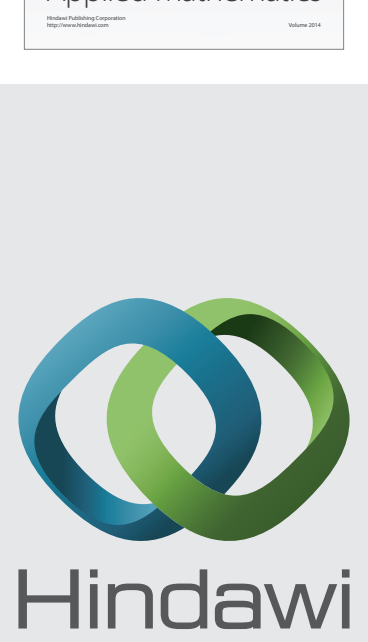

Submit your manuscripts at http://www.hindawi.com
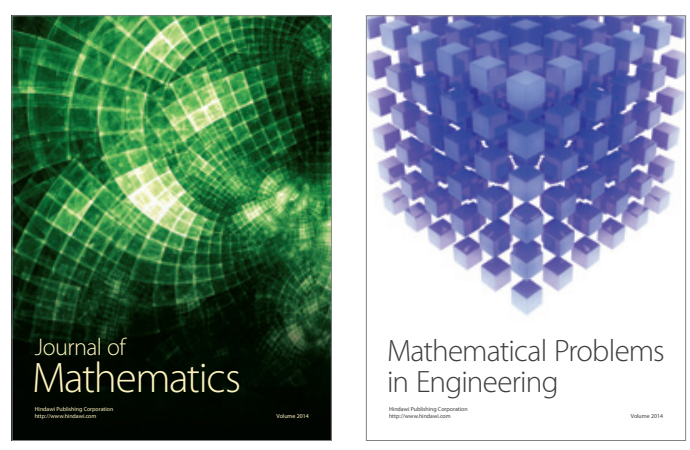

Mathematical Problems in Engineering
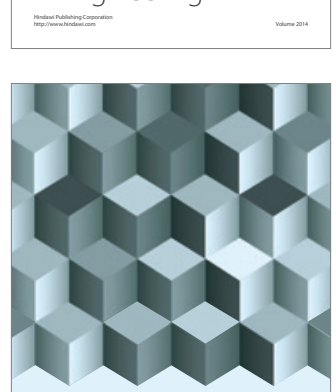

Journal of

Function Spaces
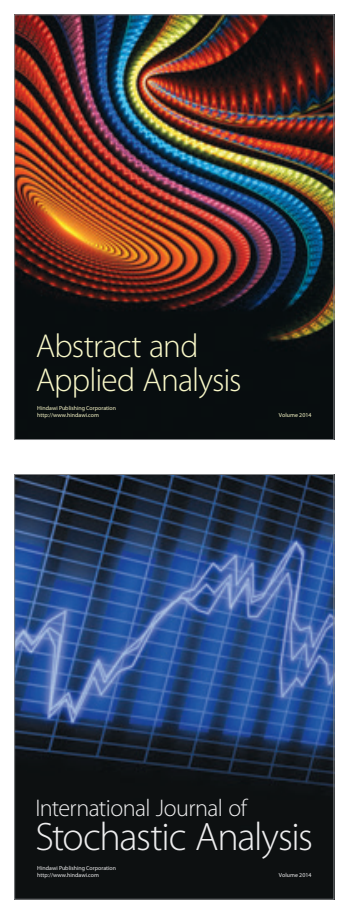

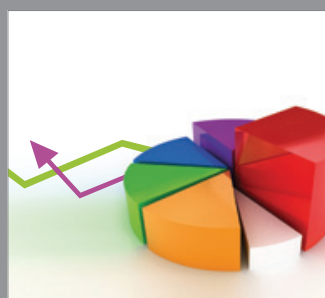

ournal of

Probability and Statistics

Promensencen
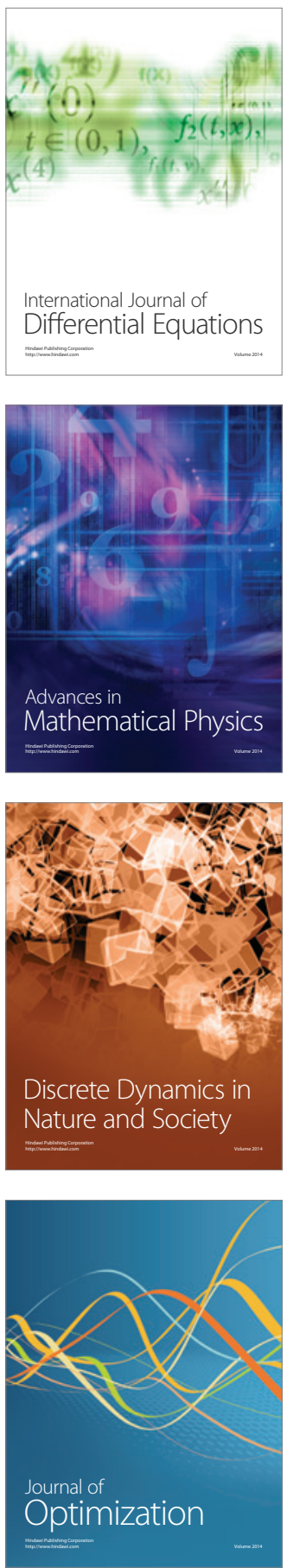